$\overline{A E E T}$

ASOCIACIÓN ESPAÑOLA

DE ECOLOGÍA TERRESTRE

\title{
Lista de revisores de la revista ECOSISTEMAS 2013
}

\author{
L. Jiménez-Eguizábal|1,* \\ (1) Revista ECOSISTEMAS. Asociación Española de Ecología Terrestre (AEET), Departamento de Biología y Geología, Universidad Rey Juan Carlos, \\ c/ Tulipán s/n, 28933 Móstoles, Madrid, España.
}

* Correpondencia: Revista Ecosistemas [revistaecosistemas@aeet.org]

Jiménez-Eguizábal, L. 2014. Lista de revisores de la revista ECOSISTEMAS 2013. Ecosistemas 23(1):64. Doi.: 10.7818/ECOS.2014.23-1.10

La labor de los revisores es fundamental para que una publicación científica realice su labor de manera adecuada y cumpla con sus objetivos de calidad. Estas personas contribuyeron generosamente revisando los manuscritos recibidos en nuestra revista durante el año 2013. ECOSISTEMAS agradece de forma muy especial su desinteresada y valiosa colaboración.

Abdelaziz, Mohamed

Agüera Buendía, Estrella

Alcorlo, Paloma

Alonso, Álvaro

Altamirano, Adison

Alviar Ramírez, Mauricio

Ameztegui, Aitor

Aragón, Pedro

Arim, Matias

Arroyo, Beatriz

Asensio Fandiño, Verónica

Baraibar, Bárbara

Baraza Ruiz, Elena

Barbeito, Ignacio

Barrios, Edmundo

Bastida, Fernando

Benito, Blas M.

Blanco, Juan A.

Blanco, Saul

Buscardo, Erika

Campos, Raquel

Cañellas, Isabel

Capdevila-Argülles, Laura

Carreira de la Fuente, José A.

Carro, Francisco

Castellanos, María Clara

Cayuela, Luis

Chacón Labella, Julia

Cortina, Jordi

Cuellar, Mamen

Damasceno, Elis

de la Cruz Rot, Marcelino de la Iglesia, Rodrigo

de la Riva, Ignacio

de Ron, Antonio

Dellafiore Capiello, Claudia M.

Díaz Ruiz, Francisco

Díaz, Marío

Domínguez, M. Teresa

Duarte, Jesús

Ebensperger, Luis

Estay, Sergio

$\mathrm{Fa}$, John

Farfán, Miguel Ángel

Felicísimo, Ángel Manuel

Fernández-Quintanilla, Cesar

Ferreras, Pablo

García Estringana, Pablo

Gómez Sal, Antonio

Gonhi, Daniel

Gonzalez-Voyer, Alejandro

Gosa, Alberto

Guadalupe Rivera, Marta

Guitart, Raimon

Guzman, David

Herrera, José Manuel

Herrero, Asier

Hidalgo, Hugo G.

Lavín González, Santiago

León Vizcaíno, Luis

Linares, Juan Carlos

Lozano, Jorge

Lucio, Antonio

Macia, Manuel
Malo, Aurelio F.

Mandujano Rodriguez, Salvador

Martin Alcón, Santiago

Rodríguez Teijeiro, José D.

Martínez de Fabricius, Ana Luján Rojas, Raquel

Martinez, Pablo Ariel

Mas, Julio

Meli, Paula

Merino, Luis

Montes Pita, Fernando

Morales, Manuel

Moreno Rueda, Gregorio

Moya Navarro, Daniel

Navas, Alfonso

Naya, Daniel

Negro Balmaseda, Juan José

Nerida, Julieta

Ochoa Gaona, Susana

Pausas, Juli G.

Perea, Ramón

Pérez, Ignacio

Pérez, Rosario

Pérez Fernández, María

Pérez Jiménez, Jesús María

Polo Cavia, Nuria

Poyatos, Rafael

Puerta-Piñero, Carolina

Puigcerver Oliván, Manel

Quesada Montano, Beatriz

Quirici, Verónica

Ramos-Jiliberto, Rodrigo

Rey-Benayas, José María

Rincón, Ana
Rodríguez, Itziar

Rodríguez-Echeverría, Susana

Rueda, Marta

Sánchez Prieto, Cristina

Sánchez Salguero, Raúl

Sánchez, Sara

Santos, Tomás

Sanz, Ma Jesús

Sevilla, Eduardo

Tejedo, Pablo

Tellería, José Luis

Townsend, Wendy

Trujillo, Laura

Uría Díez, Jaime

Valera, Francisco

Varela, Sara

Vargas Yañez, Juan Mario

Vidal, Beatriz

Videla, Cecilia

Viejo García, Rosa María

Vilagrosa, Alberto

Villafuerte, Rafael

Villalobos, Fabricio

Zafra Calvo, Noelia

Zahawi, Zak

Zamora, Regino

Zamorano-Elgueta, Carlos 